\title{
The Use of Digital Imaging Techniques for Better Understanding of Cellular Reactivity
}

\author{
Ahed J Alkhatib* \\ Department of Legal Medicine, University of Science and Technology, Jordan \\ *Corresponding author: Ahed J Alkhatib, Department of Legal Medicine, Toxicology of Forensic Science and Toxicology, School of \\ Medicine, Jordan University of Science and Technology, Jordan \\ To Cite This Article: Ahed J Alkhatib.The Use of Digital Imaging Techniques for Better Understanding of Cellular Reactivity. Am J Biomed Sci \& \\ Res. 2019 - 4(2). AJBSR.MS.ID.000780. DOI: 10.34297/AJBSR.2019.04.000780
}

Received: : July 02, 2019 | Published: July 25, 2019

\begin{abstract}
Using ordinary techniques in viewing microscopic activities is limited by being qualitative in nature. Looking for more advanced understanding of image properties suffers from subjective views. Looking for more professional imaging analysis is available and not affordable in all settings. From our experience in immunohistochemistry, we used to localize biomarkers using indirect immunoproxidase techniques, but analyzing sections was a problematic and mainly beneficial for diagnosis since the result is mainly interpreted as positive or negative. The research needs in histopathology require more data for statistical analysis through examining hypotheses. To overcome these difficulties, we used the imaging analysis tools exist in adobe Photoshop software. The idea depends in calculating the total pixels per image, then an option to select a color of the image to compute its distribution as pixels. The obtained number is divided by the total pixels in slide. The resulting number is recorded. The same process is repeated for all sections that represent study groups. Using this technique leads to generate digital data that can be statistically analyzed and to compare the differences in groups and to determine the significance in occurring of cellular phenomena. Finally, we have used this technique for more than 10 years and published important articles in various journals.
\end{abstract}

Keywords: Digital image; Adobe Photoshop; Histopathology; Immunohistochemistry; Biomarkers

\section{Introduction}

Immunohistochemistry (IHC) is used to determine both of the location and spreading of tissue antigens in cells and/or tissues via staining with specific antibodies. Indirect immunohistochemistry is applied through conjugation of secondary antibodies and streptavidin conjugated peroxidase following the incubation with primary monoclonal antibody [1-3]. In direct immunohistochemistry, the antibody is conjugated to either a fluorescent or enzymatic label. Through microscopic examination, the labeled antibodies identify the location of the position of the target antigen [4]. IHC has the advantage over other molecular and cellular techniques in its ability to visualize tissue antigens where they are expressed and distributed.

\section{Approaches for Interpretation of Immunohistochemistry Images}

IHC involves several steps, and each step is important in final staining result. It is crucial to standardize each step [5-7]. During examination of immunohistochemistry slides, there are some considerations to be taken into account inclu ding setting up a scoring system of tissue lesions. This implies determining lesion related parameters to help in constructing scoring systems. However, as far as the scoring system is clear as it helps in understanding data. The inherent problem is the lack of interpretation consistency.

The idea of using semi-quantitative scoring systems is widely accepted by researchers to make objective data from perceived IHC expression of biomarkers to help in statistical analysis and reaching the appropriate conclusions. Lack of scoring systems implies the use of general adjective words such as strong, weak, and some modifying words including more, or less [8]. The process of establishing semi-quantitative scoring systems is based on using various variables that are individually computed employing ordinal scales and then converted into a total score. The scores of different study groups can be compared using appropriate statistical tests [9]. Researchers have only set up the golden standard in IHC for the assessment of three biomarkers: estrogen receptor (ER), progesterone receptor, and Her2/neu [10].

However, there are various approaches for scoring systems in literature, and we will describe them to compare our system at the end. 


\section{Assessment of the number of positively cells stained by IHC}

This is the simplest approach in which researchers compute the total number of positively stained cells in each slide for each biomarker under study among different study groups [7,11,12].

\section{Assessment of ratio of IHC-positively stained cells and/ or area}

This approach depends on counting the percentage of positive cells stained by IHC by the number of total cells per selected area [13]. It is possible to automate this approach [14].

\section{Qualitative scoring}

Qualitative interpretation of IHC slides is widely used among researchers who range the score from negative to positive [15-17].

\section{Combination of semi-quantitative scoring systems}

This approach depends on combination existing scoring system with each other to obtain a new one. Multi-parameter scoring systems within the semi-quantitative approach are involved. According to the degree of categorizing potential, parameters under study take values ranging from 0-4, and may reach 18 [18].

\section{Assessment of objective parameters}

In this approach, scientists have tried to minimize as much as possible the subjectivity in previous approaches. Objective parameters such as optical density of positively IHC stained areas are calculated and scored objectively [14]. Data of measured objective parameters can be presented as means and standard deviations. This approach minimizes the subjectivity judgment. This approach, on the other hand, suffers from high consumption of observer's time. Usually, there is a need to use personal computers supplied with special analytical software. However, although the automation covered most areas in IHC, interpretation and results analysis are still far away from automation $[14,19]$.

As it can be seen from the above section, no approach is ideal to interpret the result of IHC, and each approach has its limitation. We have previously generated our special approach and use it in various publications.

\section{Our Experience in Developing our Approach}

We have set up three objectives in developing this approach: practical approach (easy to be applied), obtained results are reproducible and easily documented, and not expensive which means can be used by any one at any place.

To achieve these objectives, we used adobe Photoshop, and started with version 7. The first step in this approach is to create a file of photos for slides stained with IHC. These slides should be categorized within the file according to study groups. The adobe Photoshop was used to analyze the IHC photos. We opened the photos using adobe Photoshop, and adobe Photoshop gives the total pixels of the whole photo. Using another option in adobe Photoshop, we can select the color representing the biomarker under study. In our studies, we used the chromogen diaminobenzidine which gives a brown color for immunostaining. Accordingly, we used the selection option for the brown color. In this context, we see the best selection that represents the immostaining in the whole photo, which will be also computed in pixels. Now, we divide the pixels of selected color by the total pixels of the photo. From each photo, we measure 10 fields, and this process is repeated for photos. Using adobe Photoshop gives two advantages at the same time, intensity and percentage of positivity. All data are entered into statistical software programs such as excel and SPSS for analysis. According to study objectives, statistical analytic parameters such as independent T test, One Way Anova, and others can be used to test relations between variables and study hypotheses.

Using this technique, we conducted several studies and examined the impact of exercise on the outcome of Parkinson disease [20]. In a recent study, our team constructed a kinetic model to predict the occurrence and outcome of acute myeloid leukemia based on the expression of p53 and WT1 [21,22].

\section{Conclusion}

Although IHC is a very important staining technique to visualize and localize targeted antigens, it suffers from subjectivity when it comes to analyzing and interpretation of the results. Although several approaches have already been proposed to quantitative the results of IHC, but no ideal approach has been recognized so far. In this study, we introduced a new approach to participate in minimizing the subjectivity associated with IHC through using the adobe Photoshop. We have used it over 10 years in our laboratory and was shown to be stable with good results.

\section{References}

1. Ahed Al-khatib (2013) Co-expression of iNOS and HSP70 in diabetes type 1 makes a rational hypothesis to explain the diabetic neuropathy. European Scientific Journal 9(3): 145-156.

2. Ahed AlKhatib, Fatima Laiche, Mosleh Alkhatatbeh, AliyuMaje Bello, Ibrahim Ahmad Muhammad et al. (2014) Leaf extract of U. Pilulifera down regulates the expression of INOs in kidneys of diabetic rats. European Scientific Journal 10(21): 302-309.

3. Al-Jarrah M, Matalka I, Aseri HA, Mohtaseb A, Smirnova IV, et al. (2010) Exercise training prevents endometrial hyperplasia and biomarkers for endometrial cancer in rat model of type 1 diabetes. J Clin Med Res 2(5) :207-214.

4. Hui Zhang, Wen Wen, Jizhou Yan (2017) Application of immunohistochemistry technique in hydrobiological studies. Aquaculture and Fisheries 2(3): 140-144.

5. Dunstan RW, Wharton KA, Quigley C, Lowe A (2011) The use of immunohistochemistry for biomarker assessment-can it compete with other technologies? Toxicol Pathol 39(6): 988-1002.

6. Nickolay Fedchenko, Janin Reifenrath (2014) Different approaches for interpretation and reporting of immunohistochemistry analysis results in the bone tissue - a review. Diagn Pathol 9: 221.

7. Shi S-R, Shi Y, Taylor CR (2011) Antigen retrieval immunohistochemistry review and future prospects in research and diagnosis over two decades. J Histochem Cytochem 59(1): 13-32.

8. Sojo K, Sawaki Y, Hattori H, Mizutani H, Ueda M (2005). Immunohistochemical study of vascular endothelial growth factor (VEGF) and bone morphogenetic protein-2, -4 (BMP-2, -4) on lengthened rat femurs. J Cranio-Maxillo-fac Surg 33(4): 238-245.

9. Klopfleisch R (2013) Multiparametric and semiquantitative scoring systems for the evaluation of mouse model histopathology-a systematic review. BMC Vet Res 9:123. 
10. Fitzgibbons PL, Dillon DA, Alsabeh R, Berman MA, Hayes DF, et al. (2014) Template for reporting results of biomarker testing of specimens from patients with carcinoma of the breast. Arch Pathol Lab Med, 138(5): 595-601.

11. Yan Y, Gong P, Jin W, Xu J, Wu X, et al. (2012) The cell-specific upregulation of bone morphogenetic protein-10 (BMP-10) in a model of rat cortical brain injury. J Mol Histol 43: 543-552.

12. Ishihara Y, Ueki K, Sotobori M, Marukawa K, Moroi A (2014) Bone regeneration by statin and low-intensity pulsed ultrasound (LIPUS) in rabbit nasal bone. J Cranio-Maxillo-fac Surg 42(3): 185-193.

13. Wang C-J, Huang K-E, Sun Y-C, Yang Y-J, Ko J-Y, et al. (2011) VEGF modulates angiogenesis and osteogenesis in shockwave-promoted fracture healing in rabbits. J Surg Res 171: 114-119.

14. Varghese F, Bukhari AB, Malhotra R, De A (2014) IHC Profiler: an open source plugin for the quantitative evaluation and automated scoring of immunohistochemistry images of human tissue samples. PLoS One 9(5): e96801.

15. De Oliveira Neto PJ, Cricchio G, Hawthorne AC, Okamoto R, Sennerby $\mathrm{L}$, et al. (2012) Tomographic, histological, and immunohistochemical evidences on the use of N-butyl-2-cyanoacrilate for onlay graft fixation in rabbits. Clin Implant Dent Relat Res 14: 861-871.

16. Ida CM, Vrana JA, Rodriguez FJ, Jentoft ME, Caron AA, et al. (2013) Immunohistochemistry is highly sensitive and specific for detection of BRAF V600E mutation in pleomorphic xanthoastrocytoma. Acta Neuropathol Commun 1:20.

17. Sand JP, Kokorina NA, Zakharkin SO, Lewis JS, Nussenbaum B (2014) BMP-2 expression correlates with local failure in head and neck squamous cell carcinoma. Otolaryngol Head Neck Surg 150: 245-250.

18. Thoolen B, Maronpot RR, Harada T, Nyska A, Rousseaux C, et al. (2010) Proliferative and nonproliferative lesions of the rat and mouse hepatobiliary system. Toxicol Pathol, 38(7 Suppl): 5S-81S.

19. Prichard J, Bitting A, Myers J (2011) Overview of Automated Immunohistochemistry. In Handb Pract Immunohistochem, Edited by Lin F, Prichard J, Springer, New York, USA, pp. 23-30.

20.Al-Jarrah M, Obaidat H, Bataineh Z, Walton L, Al-Khateeb A (2013) Endurance exercise training protects against the upregulation of nitric oxide in the striatum of MPTP/probenecid mouse model of Parkinson's disease. Neuro Rehabilitation 32(1): 141-147.

21. Mohammad A Bani-Ahmad, Suleimman A Al-Sweedan, Mohammad A Al-Asseiri, Ahed J Alkhatib (2018) A Proposed Kinetic Model for the Diagnostic and Prognostic Value of WT1 and p53 in Acute Myeloid Leukemia. Clinical laboratory 64(3): 357-363.

22. Shi W, Gong P, Fan J, Yan YH, Ni L, et al. (2012) The expression pattern of ADP-ribosyltransferase 3 in rat traumatic brain injury. J Mol Histol 43(1): 37-47. 